
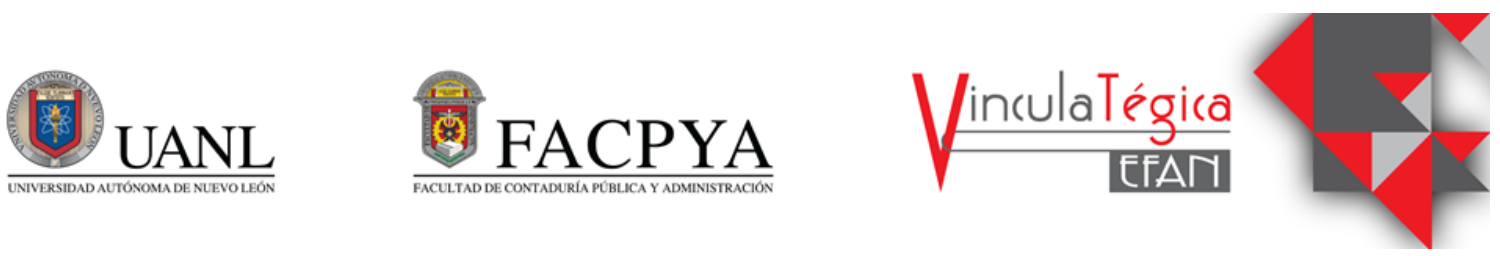

\title{
Efecto del modelo e-servicescape sobre el comportamiento eWOM, la lealtad de marca y la intención de compra en línea a través de la confianza en el sitio web en la industria de supermercados
}

\author{
Mónica Guevara Rodríguez ${ }^{1}$ \\ ${ }^{1}$ Universidad Autónoma de Tamaulipas, a2203048018@alumnos.uat.edu.mx, Matamoros SN, Zona Centro \\ Ciudad Victoria, Tamaulipas, C.P. 87000, +52 8341184775
}

Información del artículo revisado por pares

Fecha de aceptación: junio-2021

Fecha de publicación en línea: diciembre-2021

DOI: https://doi.org/10.29105/vtga7.1-140

\section{Resumen}

Gracias al nivel de penetración del Internet y, del comercio electrónico entre los usuarios surgió la necesidad de estudiar al comprador en línea desde el punto de vista de su comportamiento y hábitos en Internet. El objetivo del presente trabajo consistió en analizar el efecto del modelo e-servicescape sobre las intenciones de comportamiento en Internet, tales como el eWOM, la lealtad de marca y, la intención de compra, utilizando como mediadora a la confianza en el sitio web, entre los compradores en línea de supermercados. Y fue a través de una investigación cuantitativa que se aplicó un cuestionario en línea a consumidores habituales de productos de supermercados localizados en la región de Tamaulipas y Nuevo León, México. Dentro de los principales resultados se concluyó que el modelo e-servicescape tiene un efecto directo sobre la lealtad de marca, la intención de compra y, el comportamiento eWOM. Sin embargo, resultó interesante encontrar que el efecto indirecto por medio de la confianza en el sitio web no pudo ser comprobado en las tres variables a explicar, por lo tanto, es que se plantearon posibles razones de lo anterior.

Palabras clave: Confianza, e-servicescape, eWOM, intención de compra, lealtad de marca.

\section{INTRODUCCIÓN}

Este trabajo de investigación trata de analizar las relaciones del consumidor y la marca en

\begin{abstract}
Thanks to the level of penetration of the Internet and e-commerce among users, the need arose to study the online buyer from the point of view of their behavior and habits on the Internet. The objective of this work was to analyze the effect of the e-servicescape model on behavioral intentions on the Internet, such as eWOM, brand loyalty and purchase intention, using trust in the website as a mediator among online supermarket shoppers. And it was through a quantitative research that an online questionnaire was applied to regular consumers of products from supermarkets located in Tamaulipas and Nuevo Leon, Mexico. The main results indicated that it was concluded that the e-servicescape model has a direct effect on brand loyalty, purchase intention and eWOM behavior. However, it was interesting to find that the indirect effect through trust in the website could not be verified in the three variables to be explained, therefore possible reasons for the above were raised, as well as future lines of research to reevaluate the hypotheses presented by the mediation relationships.
\end{abstract}

Keywords: Brand loyalty, e-servicescape, eWOM purchase intention, trust.

JEL: M31, M32, M39

Internet, por lo tanto, es que se abordan datos interesantes e importantes que justifican los motivos para centrarlo en Internet $y$, en el 
comercio electrónico en el tipo de comercio al por menor. Específicamente, se analizan los aspectos que determinan comportamientos como la lealtad de marca, la intención de eWOM (comunicación boca a boca electrónica) y, la intención de compra en línea. Esta es una investigación pertinente, es decir, busca conocer y enfrentar el problema de entender lo que sucede entre el consumidor y la marca en Internet con la finalidad de identificar y explicar los motivantes que lo conducen a convertirse en un cliente leal a la marca $\mathrm{y}$, por consiguiente, le surja al un comportamiento de intención en línea. Por otro lado, la investigación se sitúa en el territorio de Internet, el cual, presenta una importante penetración entre las personas. Además, al estar situada en este contexto; las relaciones entre el consumidor y la marca se producen a nivel mundial, por lo que este trabajo tiene una relevancia global.

Desde el punto de vista teórico, esta investigación propone ampliar e ir más allá de los modelos localizados en los estudios empíricos recientes, realizando una fusión entre las relaciones planteadas por los autores entre el consumidor y la marca en Internet. Esta investigación se lleva a cabo en un lugar distinto a las investigaciones previas, además, se aplican las relaciones entre variables en otro país a los localizados en la revisión de literatura. En lo referente al contexto, el modelo es probado en un contexto diferente, por ejemplo, los estudios recientes han investigado contextos específicos como la industria de la aviación, tiendas en línea, compañía de seguros, usuarios de sitio web Shopee, universidades, minoristas de ropa, cosméticos de lujo, entre otros.

\subsection{Internet}

El uso de Internet ha ido en aumento, de acuerdo con los últimos datos de la UIT, (2019) (Unión Internacional de Telecomunicaciones), el porcentaje de hogares con acceso a Internet a nivel mundial entre el 2002 y el 2019 se ha incrementado en $325 \%$; siendo en los países en desarrollo en donde mayor crecimiento ha tenido (914\%); mientras que en los países desarrollados se ha aumentado en $148 \%$.

La situación actual de Internet a nivel de Latinoamérica y el Caribe y, de México de acuerdo con datos publicados en Statista (Johnson, 2021; Chevalier, 2020), entre los años 2015 y 2020 México posee entre el 1.7\% y $1.8 \%$ de los usuarios de Internet en el mundo $\mathrm{y}$, respecto al total de usuarios de Latinoamérica y el Caribe ha concentrado entre el $16 \%$ y el $17 \%$ de los usuarios. Ahora bien, se presenta una comparación del porcentaje de crecimiento de usuarios entre el 2015 y el 2020, siendo que a nivel mundial el crecimiento es de $48 \%$, en Latinoamérica y el Caribe del 40\% y, en México de 39\% (muy similar al crecimiento presentado en la región). Por otro lado, en el reporte de Chevalier, (2020) se realiza un pronóstico del número de usuarios de Internet en México para los siguientes cinco años, llegando al año 2025 a 95.31 millones de usuarios, lo cual representa un crecimiento del $64 \%$ respecto al año 2015.

Asimismo, de acuerdo con datos del Censo de Población y Vivienda 2020 (INEGI, 2020), en México la población en 2020 es de $126,014,024$, por lo que comparando el número de usuarios de Internet en México es posible concluir que el $64 \%$ de la población en este país utiliza Internet. Y, respecto al año 2015, la población en México según datos del INEGI, (2015) era de 119,938,473 personas, representando los usuarios de Internet al 48\% del total. Así que, comparando ambos años, existe un aumento en este periodo de $16 \%$, es decir 6,075,551 usuarios más en cinco años.

En resumen, con los datos anteriores es posible conocer la importancia del uso de Internet entre las personas, además, se observa el nivel de crecimiento que ha tenido a lo largo del tiempo y, esto da paso al siguiente aspecto a tratar: el comercio electrónico.

\subsection{Comercio electrónico}

Gracias al grado de penetración del Internet entre las personas es que el comercio electrónico se ha convertido en parte importante de la vida moderna (Coppola, 2021) y, de especial relevancia es la industria de las ventas al por menor, al año 2016 se presentan 1.66 billones de compradores de bienes y servicios en Internet a nivel mundial en este canal, 1.92 billones en 2019 y, se 
espera que para el año 2021 sean 2.14 billones de personas es decir, del año 2016 al 2021 la tendencia indica un crecimiento de $28.91 \%$. Y, hablando de ventas Sabanoglu, (2021) establece en su reporte "Ventas globales de comercio electrónico minorista 2014-2024" que en el año 2019 las ventas exceden los 3.5 trillones de dólares en todo el mundo, en 2020 representan 4.28 trillones de dólares y, para el año 2022 se esperan 5.4 trillones de dólares en ventas, en porcentaje para el año 2022 se prevé un aumento de $54.28 \%$ tomando como base al año 2019.

También cabe señalar que las compras en línea son realizadas por medio de plataformas donde los consumidores comparan y navegan para conocer y ejecutar la compra del producto o servicio requerido (Coppola, 2021). De acuerdo con el reporte "Distribución de las compras en línea globales a julio de 2019, por canal" de Sabanoglu, (2020) se presenta que los mercados en línea concentran a la mayor parte de las compras en Internet (47\%) y, en segundo lugar, al sitio web o aplicación del vendedor minorista $(26 \%)$.

Ahora bien, hablando específicamente de México, por medio del INEGI, (2018), es posible conocer el desarrollo del comercio electrónico en este país. En su último reporte, que abarca del 2013 al 2018, se encuentra que han incrementado los valores corrientes en millones de pesos en el comercio electrónico en $130 \%$. Y, a detalle, se conoce que el comercio al por mayor ha cambiado en $152 \%$ $\mathrm{y}$, en otros servicios, se ha incrementado en 89\%. Sin embargo, en donde es mayor el crecimiento es en el comercio al por menor, con un aumento de $241 \%$.

Un dato interesante es la contribución del comercio electrónico al producto interno bruto (PIB) de México. El INEGI, (2018) muestra que en el año 2018 el comercio electrónico representa el 5\% del PIB nacional $\mathrm{y}$, de manera desglosada, otros servicios representan el $2.4 \%$, el comercio al por mayor el $1.4 \% \mathrm{y}$, finalmente, el comercio al por menor, tiene una participación de $1.2 \%$. En total, entre los años mencionados, la participación ha aumentado $71 \%$. En el comercio al por mayor, el aumento es de $87 \%$ $\mathrm{y}$, en el comercio al por menor es donde se presenta un crecimiento importante (154\%).

En síntesis, en México el comercio al por menor está teniendo un importante crecimiento dentro del comercio electrónico, por lo que a continuación se describe la composición del comercio minorista, de acuerdo con los resultados de los Censos Económicos del INEGI en el año 2018; tomando como base los datos sobre los ingresos de las actividades económicas se encuentra que el comercio al por menor en supermercados es el que mayores ingresos genera; teniendo una participación del 18\% de los ingresos totales de las actividades del comercio al por menor. En segundo lugar, aparece el comercio relacionado con gasolina y diésel; siguiendo el comercio de automóviles y camionetas nuevos.

En suma, esta investigación se lleva a cabo en el contexto del comercio al por menor en supermercados debido a la importante participación del comercio minorista en México y, se considera el comercio al por menor en supermercados al ser la principal actividad económica en cuanto a ingresos generados se refiere.

Continuando con el análisis del estado actual del comercio electrónico en este país, en el reporte "Comercio electrónico en México 2020" realizado por EcommerceDB, (2021) se presentan los siguientes datos relevantes para describir al mercado mexicano. En primer lugar, se determina que al año 2020 existen 51 millones de usuarios de comercio electrónico, es decir se cuenta con $39 \%$ de penetración de usuarios de comercio electrónico respecto al total de la población y, sobre el número de usuarios de Internet en México, la penetración del comercio electrónico es de $62.99 \%$.

Sobre el mercado, las ventas netas del comercio electrónico en el año 2019 representan \$14 billones de dólares y, la tasa de crecimiento anual compuesta (CAGR) en las ventas netas del 2019 y a lo esperado al 2024 , es de $11 \%$. Cabe mencionar que las compras se concentran principalmente en productos electrónicos y de medios de comunicación. En otro sentido las cinco 
principales tiendas en línea mayormente utilizadas en México son Amazon, Mercado Libre, Walmart, Apple y Coppel (tomando como base las ventas netas), estas tiendas representan al 50\% del comercio electrónico mexicano $\mathrm{y}$, han tenido un crecimiento al 2020 de $171 \%$ desde el año 2017 (EcommerceDB, 2021). El comprador mexicano en línea de acuerdo con el reporte de EcommerceDB, (2021) tiene un importante potencial ya que se espera que para el año 2024 sean 74 millones de usuarios del comercio electrónico, es decir un crecimiento de $45 \%$ respecto al año 2020.

Gracias a los datos que reflejan la situación del comercio electrónico en México de años anteriores, actuales y de pronósticos para los siguientes años ha sido posible contextualizar el escenario en el cual se lleva a cabo este trabajo de investigación, además, de conocer las principales características que definen al comprador en línea, así como los aspectos que se encuentran relacionados con él. Por medio de lo analizado previamente se conoce del importante crecimiento del comercio electrónico por lo que surge la necesidad de continuar estudiando al comprador en línea desde el punto de vista de su comportamiento y hábitos en Internet.

\subsection{Modelo e-servicescape}

Este es un constructo de una serie de componentes que rodean al cliente durante su experiencia de compra en línea y, que considera tres dimensiones de los sitios web: apariencia estética, diseño y funcionalidad y, la seguridad financiera (Harris \& Goode, 2010); los cuales llevan a generar confianza y lealtad, o bien intención de compra (Harris \& Goode, 2010; Tran \& Strutton, 2020).

Harris \& Goode (2010) muestran y analizan un modelo sobre las intenciones de compra, confianza y del e-servicescape en ambientes en línea, además, operazionalizan y prueban el modelo del servicescape, relacionando el constructo con la confianza y las intenciones de compra. Posteriormente, Sreejesh y Ponna, (2017) demuestran el impacto del e-servicescape sobre la lealtad al analizar los sitios web de turismo en alumnos de una escuela de negocios en India.

Coincidentemente, al buscar describir al e-servicescape y, analizar las relaciones existentes entre la confianza, actitud hacia la marca y el sitio web y, las intenciones de eWOM y de compra, Wu et al. (2017) demuestran en su investigación que las dimensiones del e-servicescape tienen un efecto sobre las actitudes del consumidor y la confianza en el sitio web y, gracias a las respuestas internas de los consumidores se influye en las intenciones de $e W O M$ y de compra.

Asimismo, Tankovic \& Benazic (2018) en su artículo analizan por medio de las dimensiones del e-servicescape el efecto de las percepciones de los consumidores sobre el valor de compra y la lealtad de marca utilizando el modelo S-O-R y, aplicando un cuestionario en línea a compradores activos en Internet concluyen que el valor de compra y la lealtad son influidos por la interpretación del consumidor del e-servicescape.

De igual importancia, con el objetivo de analizar los atributos del e-servicescape y su relación con la intención de compra utilizando el tipo de usuario de Internet como moderador, Teng et al. (2018) obtienen como resultados que, en general las dimensiones de apariencia estética e interactividad incrementan las intenciones de compra de los usuarios y, al analizar los resultados con los moderadores se encontraron diferencias.

Del mismo modo, Tran \& Strutton (2020) buscan determinar sí existe diferencia entre las publicaciones de los usuarios de email y de redes sociales en relación al $e$ servicescape de los vendedores en línea, además, de investigar sí las reseñas de los clientes influyen en las percepciones de los consumidores de los e-servicescapes de los sitios web y determinar sí las percepciones de los consumidores de los e-servicescapes se relacionan con la confianza y esta con las intenciones de $e W O M$ y lealtad de marca y, concluyen que los dos grupos analizados presentan diferencias, además, las reseñas de los clientes tienen un papel importante en el constructo del e-servicescape, y este tiene un efecto positivo en el $e W O M$ y en la lealtad de marca.

Por lo tanto, se concluye que el $e$ servicescape es un constructo que se ha 
analizado y relacionado con variables como la lealtad de marca, la intención de compra y el comportamiento eWOM en Internet. Este modelo permite conocer las percepciones de los consumidores resultantes de sus interacciones con el sitio web de compra en línea y permite también, analizar a profundidad cada uno de los componentes que influyen sobre sus intenciones. Por medio de esta investigación se presenta al mencionado modelo y, además, se analiza de forma conjunta el efecto sobre tres variables de intención de comportamiento, que sí bien se han analizado anteriormente de forma particular una o dos de ellas, en este estudio se presentan como tres variables dependientes.

El objetivo del presente trabajo consiste en analizar el efecto del $e$ servicescape sobre las intenciones de comportamiento en Internet: $e W O M$, lealtad de marca e intención de compra, por medio de la confianza en el sitio web entre los compradores en línea de supermercados.

\section{MARCO TEÓRICO}

\subsection{Modelo S-O-R}

Este trabajo de investigación se basa en el modelo S-O-R, reconocido también como de estímulo - organismo - respuesta, el cual surge para determinar los efectos del ambiente físico y social en el ser humano específicamente: en los sentimientos, la forma de realizar el trabajo y sus interacciones sociales; con el objetivo de mostrar un panorama que identifique y mida el impacto de variables en la vida diaria de las personas, así como en el comportamiento y la parte afectiva y de actitudes (Mehrabian \& Russell, 1974). A continuación, se muestra el desarrollo de este modelo hasta llegar al e-servicescape.

El modelo S-O-R se resume y comprende en dos enunciados: 1) conocer o medir la influencia del estímulo físico en las emociones y, 2) identificar el efecto del estímulo sobre diferentes comportamientos, tales como el desempeño laboral y la interacción social. La investigación de Mehrabian \& Russell (1974) ayuda a definir la relación entre las tres respuestas emocionales y las variables de estímulo y, del mismo modo, las variables mediadoras están relacionadas con la clase genérica de los comportamientos de aproximación - evitación.

Se dice que en las estrategias de mercadotecnia además de considerar las características del producto y del consumidor, es importante incluir los atributos de la tienda (Buckley, 1991). Y es en la década de los 80’s en donde es aplicado a la venta al por menor, encontrando que la atmósfera (variables dentro de la tienda) influye en los consumidores en términos de dos estados emocionales: placer y excitación; siendo estos estados mediadores en los comportamientos de las personas, explicando y pronosticando el efecto de los cambios suscitados dentro de las tiendas sobre el comportamiento de compra de los clientes (Donovan \& Rossiter, 1982). Además, independientemente de la necesidad del cliente respecto a un producto, se toma en consideración que las compras se realizan dentro de un contexto (Buckley, 1991).

Gracias a la psicología ambiental existen variables que representan a los atributos de las tiendas $\mathrm{y}$, estos atributos forman parte del entorno que acompaña al consumidor durante su compra (Buckley, 1991), encontrando variables atmosféricas que influyen en una amplia variedad de evaluaciones y comportamientos del consumidor (Turley \& Milliman, 2000). En la perspectiva atmosférica y de imagen de Turley \& Fugate (1992), la atmósfera es un componente de la imagen del vendedor de la venta al por menor, y esta atmósfera es capaz de modificar el comportamiento del consumidor. Se encuentra evidencia de que la atmósfera tiene un efecto positivo sobre el nivel de gasto de los consumidores determinando la cantidad de dinero y productos que son adquiridos en una tienda; además, que es posible inducir el comportamiento del consumidor bajo las condiciones creadas en el ambiente por parte de la gerencia (Turley \& Milliman, 2000).

Específicamente en las compras en línea, el diseño del sitio web es considerado como un elemento de relevancia en la estrategia de la venta al por menor que soporta al logro del éxito de las tiendas en línea (Mummalaneni, 2005). A su vez, existe un efecto de la atmosfera del sitio web sobre las 
actitudes, satisfacción y otros comportamientos de aproximación - evitación gracias a las emociones que las personas experimentan durante la compra en línea (Eroglu et al., 2003). El modelo S-O-R de Mehrabian \& Russell (1974) ha sido un modelo viable para medir las respuestas de los consumidores en las tiendas virtuales de los sitios web (Mummalaneni, 2005). Esto se debe a que la atmósfera de un sitio web juega un papel importante al crear reacciones positivas y a que dichas reacciones serán mayores en ciertas circunstancias (Eroglu et al., 2003).

Además, se ha investigado el efecto de las señales ambientales en línea; específicamente, las relacionadas con la marca $y$, con la calidad del sitio web sobre la intención de compra del consumidor, utilizando como variables mediadoras a la confianza y al riesgo percibido; confirmando la relación, siendo de mayor importancia la marca, más que la calidad, para determinar la intención de compra en línea (Chang \& Chen, 2008).

El modelo S-O-R ha sido utilizado para enmarcar investigaciones relacionadas con las particularidades de los sitios web, las respuestas resultantes son expresadas en emociones $\mathrm{y}$, los comportamientos de compra se derivan del proceso (Tran \& Strutton, 2020). Se han investigado las relaciones entre el ambiente físico y diferentes variables como las intenciones de compra, desempeño de las empresas, etc., utilizando al modelo S-O-R (Hightower et al., 2002).

El modelo S-O-R también sirve de base para $\mathrm{Wu}$ et al., (2017), este permite construir una cadena causal que considera a las actitudes hacia la marca y al sitio web como estados actitudinales que se le presenta a los consumidores en un sitio web. Las variables utilizadas como de organismo son la confiabilidad del sitio web y, la actitud del sitio y la marca; finalmente, las respuestas, que son la parte final de la cadena, son las intenciones de compra y de $e W O M$ (Wu et al., 2017).

\subsection{Literatura e-servicescape}

Como evolución al modelo S-O-R, nace en los años 90's el modelo de servicescape, este proviene de la necesidad de conocer el impacto de lo que rodea físicamente a empleados y consumidores para lograr las metas organizaciones y las de mercadotecnia respectivamente (Bitner, 1992). Este modelo incluye las dimensiones ambientales y dos moderadores (empleados y consumidores); así como las respuestas internas que se producen, tanto cognitivas, emocionales y psicológicas $\mathrm{y}$, finalmente, el comportamiento derivado de lo anterior, que se determina como de aproximación o evitación (Bitner, 1992).

De manera sucesiva $y$, debido a la expansión del comercio electrónico, se lleva el modelo servicescape a dicho ámbito (Harris \& Goode, 2010), denominándolo e-servicescape; en donde se propone una nueva escala para medirlo, y se plantea cómo las relaciones entre las dimensiones conducen a la confianza y, a la intención de compra. La confianza es clave en los intercambios que se dan en línea, por lo que las evaluaciones del e-servicescape, la confianza en el sitio web y las intenciones de compra se encuentran relacionadas (Harris \& Goode, 2010).

Las dimensiones del modelo son tres y, a continuación, se detalla cada una de ellas. $\mathrm{La}$ apariencia estética "se refiere a las condiciones ambientales en línea y la medida en que los consumidores interpretan el panorama de servicios como atractivos" (Harris \& Goode, 2010, p. 231), que de acuerdo con los autores incluye las subdimensiones de originalidad del diseño, la apariencia visual y el valor de entretenimiento. El siguiente componente del constructo es el diseño y funcionalidad, este incluye dos conceptos: el diseño es la "disposición, organización, estructura y adaptabilidad de los sitios web, mientras que la funcionalidad se refiere al grado en que dichos elementos facilitan los objetivos del servicio" (Harris \& Goode, 2010, p. 232), las subdimensiones en el constructo son usabilidad, relevancia de la información, personalización e interactividad. El último componente es denominado seguridad financiera que "se refiere a la medida en que los consumidores perciben a los procesos de pago y a las políticas generales de un sitio web, como seguros" (Harris \& Goode, 2010, p. 233), incluye a la seguridad percibida $\mathrm{y}$ a la facilidad de pago. 
Cada uno de los componentes se evalúa respecto a la confianza en el sitio web a fin de identificar la existencia de una relación positiva y, a su vez conocer la relación de la confianza con la intención de compra, llegando a la conclusión que los tres componentes están relacionados positivamente con la confianza y, que la confianza en el sitio web determina la intención de compra.

Sobre el servicescape de Bitner (1992) se concluye se ha aplicado por Harris y Goode (2010) y reemplazan la tercera dimensión relacionada con los signos y símbolos, por la seguridad financiera. Además, es importante mencionar que al conceptualizar al e-servicescape como un constructo que incluye tres dimensiones $y$, que a su vez cada una de las dimensiones tiene subdimensiones, es posible lograr un modelo amplio y profundo (Harris \& Goode, 2010).

El e-servicescape como se ha mencionado es una aplicación del modelo $\mathrm{S}$ O-R (Harris \& Goode, 2010) y ha sido utilizado para explicar comportamientos de los consumidores en Internet, comprobándose su efecto sobre las intenciones (Tran \& Strutton, 2020; Teng et al., 2018; Tankovic \& Benazic, 2018; Wu et al., 2017; Bello et al., 2020; Sreejesh \& Ponnam, 2017).

En síntesis, con el análisis interior es posible plasmar en el modelo de la Figura 1 (Anexos) las relaciones entre las variables que son planteadas. Estableciendo y siguiendo la guía del modelo S-O-R, se determina al modelo de e-servicescape como el estímulo, a la confianza en el sitio web como el organismo $\mathrm{y}$, la respuesta representa a las intenciones de comportamiento: lealtad de marca, intención de compra y el comportamiento eWOM. Buscando identificar tanto los efectos indirectos, como los directos entre el $e$ servicescape y las variables de comportamiento.

\subsection{Hipótesis}

En seguida, se presentan las hipótesis de la investigación. Específicamente sobre la relación del modelo e-servicescape con la confianza se menciona que Harris \& Goode (2010) utilizan esta variable como mediadora para determinar la intención de comportamiento $\mathrm{y}$, los autores concluyen en su investigación que la confianza es importante en la explicación de estas relaciones en Internet. Por otro lado, Wu et al (2017) identifican que el e-servicescape impacta sobre la confianza en el sitio web y, finalmente Tran \& Strutton (2020) explican la relación entre el e-servicescape y su efecto en la confianza. El constructo de e-servicescape reúne una serie de aspectos relacionados con el sitio web, estos aspectos permiten la formación de las subdimensiones y dimensiones que son parte de él y, gracias a la percepción de los usuarios del comercio electrónico, es que generan en el consumidor la confianza en el sitio web. Por lo tanto, en esta investigación se considera a la confianza en el sitio web para mediar la influencia del modelo $e$-servicescape sobre ella. Esto lleva a la formulación de la siguiente hipótesis:

H1: el modelo e-servicescape tiene un efecto sobre la confianza en el sitio web.

E-servicescape y lealtad de marca, esta es una relación que se ha encontrado en la literatura $\mathrm{y}$, gracias al modelo $e$ servicescape se ha comprobado que por los aspectos que lo integran, originan en las personas la respuesta de la lealtad, que es un resultado deseable por parte de las marcas. Aunque es en el ambiente físico, Hightower et al (2002) encuentran un efecto positivo sobre las intenciones de comportamiento incluida la lealtad. Hablando del ambiente digital, Harris \& Goode (2010) identifican que el modelo influye sobre la lealtad, lo cual coincide con lo concluido por Sreejesh \& Ponnam (2017): el e-servicescape afecta a la e-lealtad (lealtad electrónica). Además, Tankovic \& Benazic (2018) demuestran que las dimensiones del $e$ servicescape de diseño y funcionalidad y, las de seguridad financiera, establecen el valor percibido de las compras electrónicas, lo cual afecta a la lealtad actitudinal. Asimismo, Tran $\&$ Strutton (2020) establecen que el $e$ servicescape a través de la confianza influye de manera positiva en la lealtad. En esta investigación se determina analizar el efecto e-servicescape sobre la lealtad de marca, identificando sí por medio de la confianza del sitio web es mejor explicada o bien de manera directa. 
H2a: el e-servicescape tiene un efecto sobre la lealtad de marca a través de la confianza en el sitio web.

$\mathrm{H} 2 \mathrm{~b}$ : el e-servicescape tiene un efecto directo sobre la lealtad de marca.

La intención de compra es una variable del comportamiento del consumidor que también se pretende explicar y, siendo que el e-servicescape es un modelo cuyas dimensiones conciben ciertos comportamientos como respuesta, es que se incluye a la intención de compra como una variable a explicar a través del constructo. Harris \& Goode (2010) encuentran en su investigación que el e-servicescape es un modelo que tiene influencia sobre la intención de compra. Al igual que con el tema de la lealtad Hightower et al (2002) establecen que este constructo tiene un efecto positivo sobre las intenciones de comportamiento, en este caso la intención de compra. Además, Wu et al (2017) concluyen en su trabajo que gracias a las respuestas internas del individuo se produce la influencia sobre la intención de compra.

Ya que ha sido demostrada la relación entre el e-servicescape y la intención de compra, se formula la siguiente hipótesis que establece el impacto del modelo sobre el comportamiento del consumidor y, además, el efecto de la variable confianza en el sitio web como mediadora en la relación. También se evalúa el efecto directo del modelo sobre la intención de compra.

H3a: el e-servicescape tiene un efecto sobre la intención de compra a través de la confianza en el sitio web.

$\mathrm{H} 3 \mathrm{~b}$ : el e-servicescape tiene un efecto directo sobre la intención de compra.

Finalmente se analiza el efecto del modelo e-servicescape sobre el comportamiento de intención de eWOM del consumidor. Tal como se ha mencionado con la lealtad de marca y la intención de compra, el comportamiento eWOM es también una intención que se espera que surja en el consumidor gracias a las dimensiones que componen al e-servicescape. Tran \& Strutton (2020) identificaron que por medio de la confianza en el sitio web, el e-servicescape influye de manera positiva sobre una intención de eWOM. Así como Wu et al (2017), determinaron que el eWOM es influido por las respuestas internas del individuo que son resultado de los estímulos del e-servicescape. Y, Hightower et al (2002) igualmente confirma que el e-servicescape tiene un efecto positivo sobre las intenciones de comportamiento. Por lo tanto, se define la siguiente hipótesis que muestra la relación entre el modelo y la intención de $e W O M$ en Internet, tanto de manera directa como por medio de la confianza.

H4a: el e-servicescape tiene un efecto sobre la intención de $e W O M$ a través de la confianza en el sitio web.

H4b: el e-servicescape tiene un efecto directo sobre la intención de $e W O M$.

\section{MÉTODO}

Esta es una investigación con enfoque cuantitativo, así que fue empleado el método de encuesta para la obtención de los datos. Los datos que se utilizaron en la investigación fueron conseguidos gracias a un cuestionario desarrollado y validado por Harris \& Goode, (2010), lo correspondiente al eWOM por Okazaki, (2009) y Verhoef et al., (2002) y, sobre la variable lealtad se consideró a Beatty et al., (1996). Previamente este cuestionario fue aplicado en la reciente investigación de Tran \& Strutton, (2020). Otro rasgo que se resalta es que para el levantamiento de los datos fue utilizado un cuestionario en línea por medio de la aplicación Formularios de Google.

Como fue mencionado en la sección de introducción, este trabajo se enfoca en las ventas en línea de supermercados, así que tomando esto en cuenta, la investigación se centró en la región de Nuevo León y Tamaulipas especialmente, ciudades donde es ofertado este servicio $\mathrm{y}$, es en la Tabla 1 (Anexos) donde se presenta el desglose de las ciudades en donde fue aplicado el cuestionario.

El tamaño de muestra fue de 200 personas, lo cual se obtuvo considerando una población infinita, un nivel de confianza de $95 \%$ y un margen de error de $7 \%$. De los cuales el $39.5 \%$ mencionó no haber comprado productos de supermercados en línea, resultando que el $60.5 \%$ sí lo había realizado. 
La unidad de análisis se define como consumidores regulares de productos de supermercados en la región de Nuevo León y Tamaulipas. La edad promedio de los participantes en la investigación fue de 37.96 años, teniendo una edad mínima de 20 años y una edad máxima de 74 años. Y, para conocer mejor la muestra, su composición se resume en la Tabla 2 (Anexos).

Esta investigación emplea un método de muestreo por conveniencia del tipo bola de nieve; al ser una encuesta en línea los participantes compartieron el cuestionario por Internet a posibles respondientes, quienes debían cumplir como filtro ser compradores de productos de supermercados. El levantamiento de los datos se llevó a cabo del 1 al 31 de marzo de 2021.

El análisis de las variables se realizó buscando resolver cada una de las hipótesis de la investigación. Además, ya que en la literatura se establece una relación de mediación, fue utilizado el macro Process añadido a SPSS; gracias a este macro fue posible obtener un análisis del efecto indirecto de la variable mediadora. Además, se llevó a cabo cada uno de los siguientes análisis utilizando el software SPSS. Antes, se considera pertinente mencionar que las respuestas de las preguntas establecidas en escala de Likert fueron procesadas a través de los promedios de los ítems.

* Regresión lineal simple: se realizó el análisis del efecto del e-servicescape sobre la confianza; asimismo el efecto del $e$ servicescape sobre cada una de las variables de intención de comportamiento (lealtad de marca, eWOM e, intención de compra). Además, fueron establecidos los coeficientes de regresión y, se contemplaron los datos del nivel de significancia e intervalos de confianza.

* Correlación: se realizó un análisis de correlación entre la totalidad de las variables para conocer la relación existente entre ellas. Fue identificado el coeficiente de correlación $\mathrm{y}$, su correspondiente nivel de significancia, además, de determinar el tipo de relación entre las variables (positiva o negativa).

Para la realización de la investigación fueron consideradas 58 variables de las cuales el $89.7 \%$ son variables métricas mientras que, el $10.3 \%$ restante son no métricas. Ahora bien, sobre las escalas de medición de las variables el $84.5 \%$ se encuentran dentro de la escala de intervalo, siendo este tipo de escala la de gran mayoría, seguida la escala nominal con $10.3 \%$ de las variables y, finalmente el 5.2\% son medidas en escala de razón. Específicamente, para la medición de las variables de intervalo se utilizó a la escala de Likert de cinco puntos.

\section{RESULTADOS}

4.1. Relación confianza en el sitio web modelo e-servicescape.

En primer lugar, se evaluó el efecto del modelo del e-servicescape sobre la variable confianza en el sitio web. Para esto se realizó un análisis de regresión simple utilizando como variable dependiente a la confianza y, al constructo de e-servicescape como la variable independiente. El análisis de varianza permitió conocer la prueba de significatividad $\mathrm{y}$, se identificó el ajuste del modelo a los datos. Se concluyó de acuerdo con el estadístico $\mathrm{F}$ (237.965) mayor a $1 \mathrm{y}$, la significancia (.000) menor a .05 que, se rechazó la hipótesis nula (ausencia de efecto), por lo que con este análisis se menciona que el modelo de eservicescape tiene un efecto sobre la confianza en el sitio web. Sin embargo, se continúa el análisis considerando otros datos.

A continuación, se analizó el resultado de la correlación entre ambas variables. El coeficiente de correlación fue de .816 , el cual es mayor a .5 por lo tanto se concluyó que representó un efecto grande. Se confirmó, además, por medio de la prueba de significancia que arrojó un coeficiente de .000 (mayor a .05). La correlación mostró una relación positiva entre la confianza en el sitio web y el modelo e-servicescape, es decir, a medida que la percepción en el modelo de $e$ servicescape aumenta, la confianza en el sitio web se incrementa proporcionalmente. Por otro lado, es importante mencionar que el coeficiente de determinación $\left(\mathrm{R}^{2}\right)$ fue de $66.7 \%$ así que, la variabilidad compartida entre ambas variables fue de dicho porcentaje.

Hasta este punto se concluyó que el modelo propuesto es significativamente bueno para predecir la variable, sin embargo, es importante la evaluación de la contribución de 
la variable independiente, representada por el constructo $e$-servicescape sobre la confianza en el sitio web. Se determinó por medio del coeficiente $\beta 1$ (1.392) que el e-servicescape tuvo un impacto positivo sobre la confianza en el sitio web ya que fue diferente a cero $y$, mayor al error estándar.

Enseguida se realiza el análisis de significatividad, al comparar la significancia del modelo (.000) con .05 se concluyó que existe casi nula probabilidad de que tenga ausencia del efecto, por lo tanto, es posible afirmar que el modelo e-servicescape tuvo un efecto sobre la confianza en el sitio web, incluso se menciona que este efecto es positivo.

\subsection{Relación lealtad de marca - modelo $e$ - servicescape}

* H2a: el e-servicescape tiene un efecto sobre la lealtad de marca a través de la confianza en el sitio web.

Gracias al análisis del efecto indirecto del e-servicescape a través de la variable mediadora (confianza) sobre la lealtad de marca, se concluyó que el coeficiente de regresión fue de .2723 , y, se puede decir que el efecto indirecto no fue significativo porque el intervalo de confianza consideró el cero (.0969, .6971). Con el análisis anterior es posible determinar que la hipótesis que muestra la mediación de la confianza fue rechazada, no obstante, sí existe efecto directo del e-servicescape sobre la lealtad de marca.

* H2b: el e-servicescape tiene un efecto directo sobre la lealtad de marca.

Se realizó un análisis de regresión simple para conocer el efecto del modelo $e$ servicescape sobre la variable lealtad de marca de manera directa. Se definió al e-servicescape como la variable independiente y, la lealtad de marca es la variable dependiente. Se concluyó de acuerdo con el estadístico F (15.935) mayor a $1 \mathrm{y}$, la significancia (.001) menor a .05 que, se rechazó la hipótesis nula que demostró a la ausencia de efecto, así que el modelo de $e$ servicescape tuvo un efecto sobre la lealtad de marca.

En cuanto a la correlación entre el modelo e-servicescape y la lealtad de marca, el coeficiente resultante fue de .344 , el cual fue menor a .5 por lo tanto la relación tiene un efecto moderado. A pesar de tener la característica de ser moderada, a través de la prueba de significancia con un coeficiente de .000 (mayor a .05) fue posible demostrar una relación significativa. La correlación presentó una relación positiva entre la lealtad de marca y el e-servicescape.

A continuación, se realiza el análisis de los resultados de la regresión entre ambas variables. A través del coeficiente $\beta 1(.520)$ el e-servicescape tuvo un impacto positivo sobre la lealtad de marca ya que fue diferente a cero y, mayor al error estándar. Por otro lado, se comparó la significancia del modelo (.000) con .05 y se concluyó que no existe ausencia del efecto, por lo tanto, el modelo $e$ servicescape tuvo un efecto sobre lealtad de marca, además, que dicho efecto fue positivo.

\subsection{Relación intención de compra - modelo e-servicescape}

* H3a: el e-servicescape tiene un efecto sobre la intención de compra a través de la confianza en el sitio web.

Los resultados presentaron el análisis del efecto indirecto del e-servicescape por medio de la variable confianza sobre la intención de compra. Se encontró que el coeficiente de regresión es de .2264, sin embargo, se concluyó que el efecto indirecto no es significativo, ya que el intervalo de confianza contempló al valor cero (-.1499, .6520), lo cual no es aceptable. Se concluyó que es rechazada la hipótesis que establece que la confianza es mediadora en la relación entre el e-servicescape.

* H3b: el e-servicescape tiene un efecto directo sobre la intención de compra.

Para conocer la existencia del efecto directo entre el modelo de e-servicescape y la intención de compra se realizó el análisis de regresión simple entre ambas variables. La intención de compra representó a la variable a explicar y, el e-servicescape es la variable independiente del modelo, gracias al estadístico $\mathrm{F}$ (34.233) mayor a $1 \mathrm{y}$, la significancia (.000) mayor a .05 que fue rechazada la hipótesis nula (ausencia de efecto), por lo tanto, el e-servicescape tuvo un efecto sobre la intención de compra.

En seguida, se presenta la correlación entre el modelo e-servicescape y la lealtad de 
marca, el coeficiente resultante fue de .473 , el cual fue menor a .5 por lo tanto se concluyó la existencia de un efecto moderado. A pesar de tener la característica de ser moderada, a través de la prueba de significancia con un coeficiente de .000 (mayor a .05) fue posible demostrar una relación significativa. La correlación presentó una relación positiva entre la intención de compra y el $e$ servicescape.

A continuación, se realiza el análisis de los resultados de la regresión entre ambas variables. A través del coeficiente $\beta 1(.740)$ el $e$-servicescape tuvo un impacto positivo sobre la intención de compra ya que es diferente a cero y, mayor al error estándar. Por medio del análisis de regresión lineal, se comparó la significancia del modelo (.009) con .05 y se concluyó que no existió ausencia del efecto, por lo tanto, el modelo e-servicescape tiene un efecto sobre la intención de compra, además, que dicho efecto es positivo.

\subsection{Relación $e W O M-$ modelo $e$ - servicescape}

* H4a: el e-servicescape tiene un efecto sobre la intención de $e W O M$ a través de la confianza en el sitio web.

Dentro de los resultados de este análisis se encontró un análisis de regresión que considera al efecto indirecto de la confianza y, se concluyó que el coeficiente de regresión es de .6117, además, el efecto indirecto no fue significativo porque el intervalo de confianza contempló al valor cero (-.0327, 1.1988). Se encontró que la hipótesis establecida fue rechazada, por lo que la confianza en el sitio web no es mediadora en la relación entre el e-servicescape y la intención de $e W O M$.

* H4b: el e-servicescape tiene un efecto directo sobre la intención de $e W O M$.

Para conocer la existencia del efecto directo entre el modelo de e-servicescape y la intención $e W O M$ se realizó el análisis de regresión simple entre ambas variables. $\mathrm{La}$ intención de $e W O M$ representó a la variable a explicar y, el e-servicescape fue la variable independiente del modelo. Se concluyó gracias al estadístico F (39.555) mayor a $1 \mathrm{y}$, la significancia (.000) mayor a .05 que fue rechazada la hipótesis nula (ausencia de efecto), por lo tanto, el e-servicescape tuvo un efecto sobre la intención de $e W O M$.

A continuación, se presenta la correlación entre el modelo e-servicescape y la intención de $e W O M$. El coeficiente fue de .50 , el cual fue igual a .5 por lo tanto se concluyó en esta relación un efecto grande. A través de la prueba de significancia con un coeficiente de .000 (mayor a .05) fue posible demostrar una relación significativa. La correlación presentó una relación positiva entre la intención de $e W O M$ y el $e$ servicescape.

En seguida, se realizó el análisis de los resultados de la regresión entre ambas variables. A través del coeficiente $\beta 1$ (1.218) el e-servicescape tuvo un impacto positivo sobre la intención de eWOM ya que fue diferente a cero $\mathrm{y}$, mayor al error estándar. Además, se comparó la significancia del modelo (.000) con .05 y se concluyó que no existe ausencia del efecto, por lo tanto, el modelo e-servicescape tuvo un efecto sobre la intención de $e W O M$, además, que dicho efecto fue positivo.

\section{CONCLUSIONES}

A continuación, se presentan las conclusiones respecto a cada una de las hipótesis de la investigación. Respecto a la H1 se concluye que el modelo -servicescape tiene un efecto positivo sobre la confianza en el sitio. Por lo tanto, la hipótesis H1 no es rechazada. Sobre la hipótesis $\mathrm{H} 2 \mathrm{a}$, que muestra un efecto del e-servicescape sobre la lealtad de marca a través de la confianza en el sitio web, se menciona que es rechazada. Los resultados del efecto indirecto demuestran que el efecto no es significativo, contrario a la relación directa, donde el $e$ servicescape tiene un impacto sobre la lealtad de marca sin necesidad de una variable mediadora. Este es un resultado interesante ya que de alguna forma se está descartando el modelo empleado.

Por otro lado, es posible concluir que el modelo e-servicescape tiene un efecto directo sobre la lealtad de marca, por lo tanto, no es rechazada la hipótesis $\mathrm{H} 2 \mathrm{~b}$; este efecto tiene la característica de ser positivo y significativo, sin embargo, es importante ser precavidos con esta conclusión, ya que la 
relación entre ambas variables es del tipo moderada, aunque significativa.

Continuando con la respuesta a las hipótesis, se concluye que la hipótesis $\mathrm{H} 3 \mathrm{a}$ es rechazada debido a que los resultados muestran la inexistencia de un efecto significativo del e-servicescape sobre la intención de compra utilizando a la confianza en el sitio web como variable mediadora. Esta conclusión es coincidente con la planteada sobre la variable lealtad de marca, en donde se descarta lo asumido por el modelo S-O-R de utilizar una variable como mediación, no obstante, se analizarán las implicaciones de lo anterior más adelante.

Respecto a la hipótesis H3b se concluye que no es rechazada ya que se demuestra que sí existe un efecto directo significativo del e-servicescape sobre la intención de compra, además, este efecto es positivo.

Finalmente, se abordan las últimas hipótesis; en el análisis del efecto indirecto del e-servicescape sobre la intención de $e W O M$ por medio de la confianza se concluye que es rechazada la hipótesis $\mathrm{H} 4 \mathrm{a}$, así que la confianza en el sitio web no es una variable mediadora adecuada. De esta forma se deberán replantear las relaciones de mediación en este modelo y, además, definir otros aspectos para identificar la ausencia de un efecto significativo, tal como se había comprobado en estudios empíricos anteriores. Respecto a la $\mathrm{H} 4 \mathrm{~b}$, esta no es rechazada, por lo tanto, el e-servicescape tiene un efecto directo sobre la intención de $e W O M$. Este es un efecto significativo y positivo, además, de tener una alta correlación entre las variables.

La investigación desarrollada presenta una serie de limitaciones que, una vez conocidos los resultados se concluye que están relacionadas con la metodología, ya que en la totalidad de las hipótesis que tienen como objetivo probar al modelo S-O-R utilizando la mediación de la variable confianza en el sitio web, han sido rechazadas; a pesar de que en la literatura se ha demostrado su efecto. Se considera que las limitaciones van de la mano con los posibles motivos de rechazo. Sin embargo, es importante mencionar, que el constructo $e$ servicescape sí tiene un efecto sobre las diferentes variables de comportamiento en el modelo, pero no fue posible concluir la relación a través de la variable mediadora.

Las limitaciones se concentran en la metodología $\mathrm{y}$, pueden resumirse en: el tamaño de muestra; aunque se logra un tamaño de muestra de 200 personas, sin embargo, para las preguntas relacionadas con la compra en línea, la muestra es de 120 respondientes. El método de muestreo es otra de las limitantes; para elegir a los participantes se sigue el método de muestro bola de nieve, el cual es no probabilístico, así que no es posible concluir que los resultados sean estadísticamente representativos.

La ubicación geográfica es un punto importante de resaltar, la encuesta fue aplicada en ciudades del estado de Tamaulipas y, Nuevo León, sin embargo, al utilizar un método de muestreo no probabilístico, es que los respondientes se concentraron en la ciudad donde reside la mayor parte de ellos (Ciudad Victoria); pudiendo haber utilizado en su lugar un muestreo por cuotas, aunque es no probabilístico, pero sí habría asegurado una mejor distribución de la muestra por ciudad.

El factor de la pandemia también se considera como limitación, a pesar de que la encuesta se aplica en línea, es un fenómeno que limita la búsqueda de participantes de manera personal.

Y, la traducción del instrumento, se considera que debe ser mejorado, ya que durante el trabajo de campo se aclaran algunas dudas sobre las preguntas, y dichas dudas se atribuyen a la traducción realizada.

Como futuras líneas de investigación se propone, en primer lugar, redefinir el apartado de metodología, tomando en consideración los argumentos mencionados en las limitaciones, ya que se presume que con un mayor tamaño de muestra y, un muestreo que asegure la representatividad estadística, se podrán conseguir resultados más apegados al modelo teórico. Aunque, los resultados obtenidos son reveladores demostrando una relación directa significativa del e-servicescape sobre las 
intenciones de comportamiento.

En segundo lugar, es importante analizar la inclusión de otras variables mediadoras, ya sea sustituyendo a la confianza en el sitio web, o agregando otras como la satisfacción, los beneficios esperados, el apego a la marca, el compromiso del cliente, entre otras. De esta forma es posible probar el modelo e identificar sí depende de la variable mediadora elegida (s) para determinar el efecto indirecto. En tercer lugar, es sugerido utilizar variables moderadoras con la finalidad de identificar diferencias, principalmente relacionadas con el tipo de producto, por ejemplo, productos de primera necesidad y productos de lujo; además, de moderadores como la edad, género $y$, resultaría interesante identificar diferencias en el comportamiento entre quienes son nuevos usuarios debido a la pandemia y, aquellos que son usuarios experimentados en las compras en línea.

Finalmente, se propone realizar una investigación que involucre a una sola variable dependiente, de esta manera se reduce el tamaño del instrumento $\mathrm{y}$, se concentra el análisis sobre una variable de comportamiento. El estudio puede aplicarse tomando en consideración a otro contexto de compras en línea, diferente a supermercados, o bien, llevar a cabo en otra ciudad, región o país. 


\section{REFERENCIAS}

Beatty, S. E., Mayer, M., Coleman, J. E., Reynolds, K. E., \& Lee, J. (1996). Customer-sales associate retail relationships. Journal of Retailing, 72(3), 223-247. https://doi.org/10.1016/S0022-4359(96)90028-7

Bello, K.B., Jusoh, A. and Md Nor, K. (2020), "Relationships and impacts of perceived CSR, service quality, customer satisfaction and consumer rights awareness", Social Responsibility Journal, Vol. ahead-of-print No. ahead-of-print. https://doi.org/10.1108/SRJ-01-20200010

Best, R. de. (2021). Volumen total de pago de PayPal desde el primer trimestre de 2014 hasta el cuarto trimestre de 2020. Statista. https://www.statista.com/statistics/277841/paypalstotal-payment-volume/

Bitner, M. J. (1992). Servicescapes: The Impact of Physical Surroundings on Customers and Employees. Journal of Marketing, 56(2), 57. https://doi.org/10.2307/1252042

Chang, H. H., \& Chen, S. W. (2008). The impact of online store environment cues on purchase intention: Trust and perceived risk as a mediator. Online Information Review, 32(6), 818841. https://doi.org/10.1108/14684520810923953

Chevalier, S. (2020). Número de usuarios de Internet en México del 2015 al 2025. Statista. https://www.statista.com/statistics/184711/mexico-number-of-internet-users/

Clement, J. (2020). Impacto del coronavirus en el tráfico en línea de industrias seleccionadas en el $\begin{array}{lllll}\text { mundo } & a & \text { octubre } & \text { de } & \text { Statista. }\end{array}$ https://www.statista.com/statistics/1105486/coronavirus-traffic-impact-industry/

Coppola, D. (2021). Comercio electrónico mundial - Estadísticas y hechos. Statista. https://www.statista.com/topics/871/online-shopping/\#dossierSummary_chapter5

Donovan, R., \& Rossiter, J. (1982). Store atmosphere: an environmental psychology approach. Journal of Retailing, 58(1), 34-57.

EcommerceDB. (2021). Comercio electrónico en México 2020. EcommerceDB. https://www.statista.com/study/70370/ecommerce-in-mexico/

Eroglu, S. A., Machleit, K. A., \& Davis, L. M. (2003). Empirical Testing of a Model of Online Store Atmospherics and Shopper Responses. Psychology and Marketing, 20(2), 139-150. https://doi.org/10.1002/mar.10064

Harris, L. C., \& Goode, M. M. H. (2010). Online servicescapes, trust, and purchase intentions. Journal of Services Marketing, 24(3), 230-243. https://doi.org/10.1108/08876041011040631

Hightower, R., Brady, M. K., \& Baker, T. L. (2002). Investigating the role of the physical environment in hedonic service consumption: An exploratory study of sporting events. Journal of Business Research, 55(9), 697-707. https://doi.org/10.1016/S01482963(00)00211-3

INEGI. (2015). Encuesta Intercensal 2015. Instituto Nacional de Estadística y Geografía. https://www.inegi.org.mx/programas/intercensal/2015/

INEGI. (2018). Valor Agregado Bruto del Comercio Electrónico. Instituto Nacional de Estadística y Geografía. https://www.inegi.org.mx/programas/vabcoel/2018/

INEGI. (2020). Censo de Población y Vivienda 2020. Instituto Nacional de Estadística y Geografía. https://www.inegi.org.mx/programas/ccpv/2020/

J. Clement. (2021). Los sitios web minoristas en línea más populares en el mundo en 2020, por tráfico mensual promedio. Statista. https://www.statista.com/statistics/274708/onlineretail-and-auction-ranked-by-worldwide-audiences/

Johnson, J. (2021). Usuarios de internet por región. Statista. https://www.statista.com/statistics/265147/number-of-worldwide-internet-users-byregion/

Mehrabian, A., \& Russell, J. A. (1974). An approach to environmental psychology. The MIT.

Mummalaneni, V. (2005). An empirical investigation of Web site characteristics, consumer 
emotional states and on-line shopping behaviors. Journal of Business Research, 58(4), 526-532. https://doi.org/10.1016/S0148-2963(03)00143-7

Okazaki, S. (2009). The tactical use of mobile marketing: How adolescents' social networking can best shape brand extensions. Journal of Advertising Research, 49(1), 12-26. https://doi.org/10.2501/S0021849909090102

Patrick G. Buckley. (1991). An S-O-R Model of the Purchase of an Item in a Store. Advances in Consumer Research, 18, 491-500.

Sabanoglu, T. (2020a). Distribución de las compras online en el mundo a julio de 2019, por canal. Statista. https://www.statista.com/statistics/861336/share-online-shopping-customers-vssales-by-platform/

Sabanoglu, T. (2021b). Ventas minoristas de comercio electrónico en el mundo de 2014 a 2024. Statista. https://www.statista.com/statistics/379046/worldwide-retail-e-commerce-sales/

Sreejesh, S., \& Ponnam, A. (2017). Investigating the Process Through which E-Servicescape Creates E-Loyalty in Travel and Tourism Websites. Journal of Travel and Tourism Marketing, 34(1), 20-39. https://doi.org/10.1080/10548408.2015.1126213

Tankovic, A. C., \& Benazic, D. (2018). The perception of e-servicescape and its influence on perceived e-shopping value and customer loyalty. Online Information Review, 42(7), 1124-1145. https://doi.org/10.1108/OIR-12-2016-0354

Teng, H. J., Ni, J. J., \& Chen, H. H. (2018). Relationship between e-servicescape and purchase intention among heavy and light internet users. Internet Research, 28(2), 333-350. https://doi.org/10.1108/IntR-10-2016-0303

Tran, G. A., \& Strutton, D. (2020). Comparing email and SNS users: Investigating e-servicescape, customer reviews, trust, loyalty and E-WOM. Journal of Retailing and Consumer Services, 53. https://doi.org/10.1016/j.jretconser.2019.03.009

Turley, L. W., \& Fugate, D. L. (1992). The multidimensional nature of service facilities: Viewpoints and recommendations. Journal of Services Marketing, 6(3), 37-45. https://doi.org/10.1108/08876049210035926

Turley, L. W., \& Milliman, R. E. (2000). Atmospheric effects on shopping behavior: A review of the experimental evidence. Journal of Business Research, 49(2), 193-211. https://doi.org/10.1016/S0148-2963(99)00010-7

UIT. (2019). ITU Statistics 2019. Unión Internacional de Telecomunicaciones. http://www.itu.int/ict/statistics

Verhoef, P. C., Franses, P. H., \& Hoekstra, J. C. (2002). The Effect of Relational Constructs on Customer Referrals and Number of Services Purchased from a Multiservice Provider: Does Age of Relationship Matter? Journal of the Academy of Marketing Science, 30(3), 202-216. https://doi.org/10.1177/0092070302303002

Wu, W. Y., Quyen, P. T. P., \& Rivas, A. A. A. (2017). How e-servicescapes affect customer online shopping intention: the moderating effects of gender and online purchasing experience. Information Systems and E-Business Management, 15(3), 689-715. https://doi.org/10.1007/s10257-016-0323-x 


\section{ANEXOS}

Figura 1. Modelo de investigación

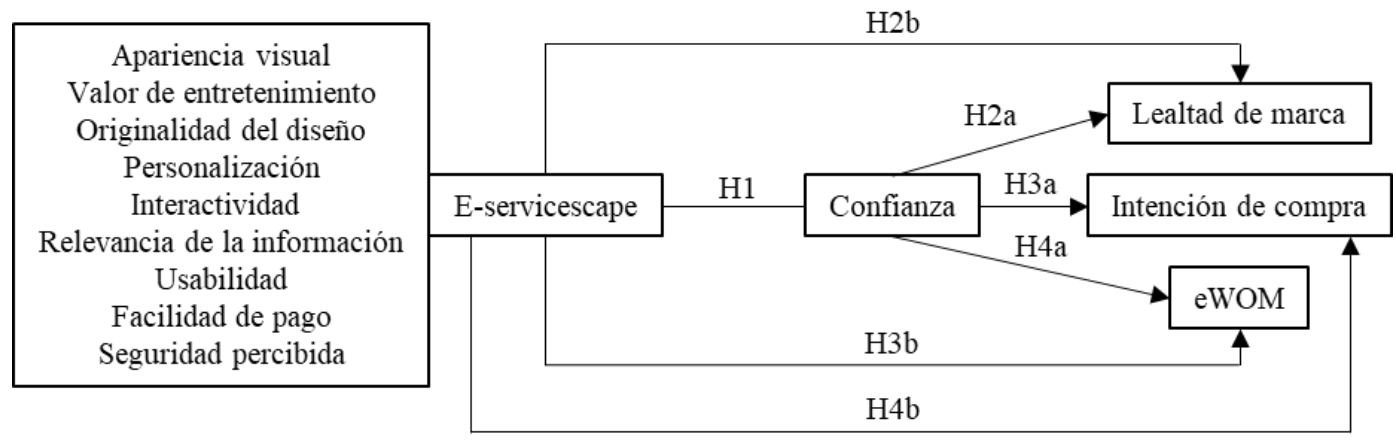

Fuente: Elaboración propia.

\section{Tabla 1}

\begin{tabular}{lcl}
\hline \multicolumn{1}{c}{ Estado } & $\%$ & \multicolumn{1}{c}{ Ciudades } \\
\hline Tamaulipas & 81.5 & $\begin{array}{l}\text { Ciudad Victoria (88\%), Matamoros (5\%), } \\
\text { Reynosa (4\%), Tampico (4\%) } \\
\text { Nuevo León }\end{array}$ \\
\hline Fuente: Elaboración propia. & 18.5 & Monterrey y zona metropolitana (100\%)
\end{tabular}

Tabla 2

\begin{tabular}{clc}
\hline \multicolumn{2}{c}{ Opción } & $\%$ \\
\hline \multirow{2}{*}{ Género } & Mujer & 73.5 \\
& Hombre & 26.5 \\
\hline \multirow{2}{*}{ ¿Tiene hijos? } & $\mathrm{Si}$ & 68.5 \\
& No & 31.5 \\
\hline & Trabajador de tiempo completo & 57.5 \\
& Ama de casa & 16.0 \\
& Negocio propio & 10.0 \\
\multirow{2}{*}{ Ocupación } & Trabajador independiente & 7.5 \\
& Maestro & 4.5 \\
& Estudiante & 4.0 \\
& Pensionado & 0.5 \\
\hline
\end{tabular}

Fuente: Elaboración propia. 